\title{
Zöliakienachweis: Den Bulbus duodeni nicht vergessen!
}

\section{Zur Diagnose der Zöliakie im Erwach- senenalter sind neben der Serologie auch histologische Untersuchungen von Biopsien aus dem distalen Duo- denum und dem Bulbus duodeni erforderlich.}

— Die Zöliakie oder glutensensitive Enteropathie ist definiert als ein Zustand einer gesteigerten Immunantwort auf aufgenommenes Gluten (zum Beispiel im Weizen, Roggen oder Hafer) bei genetisch suszeptiblen Personen. Der international vereinbarte Goldstandard für die Diagnose beinhaltet den Nachweis einer Atrophie der Mikrovilli in einer Dünndarmbiopsie. Ergänzt wird die Diagnose durch immunologische Marker wie die Gewebetransglutaminase und Endomysium-Antikörper sowie die klinische Besserung durch eine glutenfreie Diät.

Nachdem Dünndarmbiopsien nicht leicht zu gewinnen sind, hat man untersucht, ob auch distale Duodenalbiopsien ausreichen. Wegen des zumindest bei Kindern bekannten fleckförmigen Auftretens der villösen Atrophie empfehlen die Leitlinien aber mehrere Biopsien zu entnehmen. Zumindest theoretisch müssten die Gewebeveränderungen in den proximalen Abschnitten des Duodenums häufiger sein als in den distalen, da hier die Glutenbelastung am höchsten ist.

In einer prospektiven Studie wurden bei 461 Patienten im medialen Alter von 51 Jahren (126 mit neu diagnostizierter Zöliakie, 85 mit bekannter Zöliakie und 250 Kontrollen) der Antikörperstatus bestimmt und fünf Biopsien entnommen. Vier Biopsien erfolgten aus der Pars horizontalis des Duodenums, eine aus dem Bulbus duodeni. Bei 9\% der Patienten mit neu entdeckter Zöliakie und bei $14 \%$ der Patienten mit bekannter langjähriger Zöliakie fand sich nur in dem Biopsien aus dem Bulbus duodeni eine villöse Atrophie.

Beim Vergleich der histologischen Veränderungen des Bulbus duodeni mit den distalen Duodenum zeigte sich, dass 31 von 85 Patienten mit bekannter Zöliakie und 21 von 126 Patienten mit neu diagnostizierter Erkrankung eine Diskrepanz im Schweregrad der Läsionen zwischen den beiden Entnahmestellen der Biopsie aufwiesen. Bei den Kontrol- len war dies nur bei 18 von 250 der Fall. Unter den Patienten mit Diskrepanzen im Schweregrad zwischen distalem Duodenum und Bulbus hatten 24 von 31 mit bekannter Zöliakie und 16 von 21 mit neu diagnostizierter Zöliakie sogar die ausgeprägteren Läsionen im Bulbus duodeni.

\section{Kommentar}

Aufgrund ihrer Ergebnisse schlagen die Autoren vor, auf der Suche nach der villösen Atrophie bei Zöliakieverdacht immer auch Biopsien aus dem Bulbus duodeni zu entnehmen. Die Rate der richtigen Diagnosen kann auf diese Weise um etwa 10\% gesteigert werden. Manchmal sind es Kleinigkeiten, die den entscheidenden Unterschied ausmachen.

H. S. FÜESSL .
- K. E. Evans et al.
(Korres.: K.E. Evans MRCP, Room P39, Depart- ment of Gastroenterology and Liver Unit, Royal Hallamshire Hospital, Sheffield, South Yorkshire S102JF, kate.evens@sth.nhs.uk): A prospective study of duodenal bulb biopsy in newly dia- gnosed and established adult celiac disease. Am. J. Gastroenterol. 2011; 106: 1837-1842



